\title{
L-CAQ: Joint Link-Oriented Channel-Availability and Channel-Quality Based Channel Selection for Mobile Cognitive Radio Networks
}

\author{
Md. Arafatur Rahman ${ }^{\mathrm{a}, \mathrm{b}}$, A. Taufiq Asyhari ${ }^{\mathrm{c}}$, Md Zakirul Alam Bhuiyan ${ }^{\mathrm{d}}$, \\ Qusay Medhat Salih ${ }^{\mathrm{a}}$, Kamal Zuhairi Bin Zamli ${ }^{\mathrm{a}, \mathrm{b}}$ \\ ${ }^{a}$ Faculty of Computer Systems 85 Software Engineering, University Malaysia Pahang, \\ Gambang, 26300, Malaysia. \\ ${ }^{b}$ IBM Center of Excellence, University Malaysia Pahang, Malaysia. \\ ${ }^{c}$ Centre for Electronic Warfare, Information and Cyber, Cranfield University, \\ Defence Academy of the UK, Shrivenham SN6 8LA, UK. \\ ${ }^{d}$ Department of Computer and Information Sciences Fordham University, JMH 328A, \\ Bronx NY, 10458 USA.
}

\begin{abstract}
Channel availability probability (CAP) and channel quality (CQ) are two key metrics that can be used to efficiently design a channel selection strategy in cognitive radio networks. For static scenarios, i.e., where all the users are immobile, the CAP metric depends only on the primary users' activity whereas the CQ metric remains relatively constant. In contrast, for mobile scenarios, the values of both metrics fluctuate not only with time (time-variant) but also over different links between users (link-variant) due to the dynamic variation of primary- and secondary-users' relative positions. As an attempt to address this dynamic fluctuation, this paper proposes L-CAQ: a link-oriented channelavailability and channel-quality based channel selection strategy that aims to maximize the link throughput. The L-CAQ scheme considers accurate estimation of the aforementioned two channel selection metrics, which are governed by the mobility-induced non-stationary network topology, and endeavors to select a channel that jointly maximizes the CAP and CQ. The benefits of the proposed scheme are demonstrated through numerical simulation for mobile cognitive radio networks.
\end{abstract}

Keywords: Channel availability, channel quality, channel selection, cognitive radio, mobile networks, mobility.

Email addresses: arafatur@ump.edu.my (Md. Arafatur Rahman), taufiq-a@ieee.org (A. Taufiq Asyhari), qusaysalih81@gmail.com (Md Zakirul Alam Bhuiyan), qusaysalih81@gmail.com (Qusay Medhat Salih), kamalz@ump.edu.my (Kamal Zuhairi Bin Zamli) 


\section{Introduction}

Channel selection is one of the important tasks in Cognitive Radio Networks (CRNs) to enable a Cognitive User (CU) to communicate with its neighbors. A common purpose of this task is to select the "best" channel among the list of available channels that maximizes the throughput and minimizes the interference to Primary Users (PUs). In the literature there exist two prominent metrics that are widely used for channel selection, namely Channel Availability Probability (CAP) and the Channel Quality (CQ). Herein the CAP corresponds the probability of a channel licensed to a PU being available for communications of unlicensed users [1] whereas the CQ is associated with the maximum amount of data per unit time that can be reliably delivered over the given channel [21]. Accurate acquisition of these features will enable the design of throughputefficient channel selection that allows the $\mathrm{CU}$ to maximize the communication opportunities.

In static scenarios, referred to as Static CRNs (SCRNs) where all PUs and CUs are fixed and immobile, the first feature, namely CAP, depends only on the PU activity probability, i.e., the probability of the channel being occupied by the PU transmission, which can be estimated using the past channel occupancy history [1, 29]. On the other hand, the second feature, namely CQ depends on several factors such as the channel bandwidth, transmit power, overall medium attenuation and interference. In static scenario CQ remains relatively constant and similarly to CAP, the $\mathrm{CU}$ can also estimate $\mathrm{CQ}$ through the past channel history [3].

In mobile scenarios, referred to as Mobile CRNs (MCRNs) where the PUs and/or CUs are mobile, however, both the CAP and CQ vary not only with time but also with link. ${ }^{1}$ In order to better illustrate this time and link variation, consider an example of MCRNs in Fig. 1. In Fig. 1 (a), at time $t_{0}$, the $i$-th CU, $u_{i}$ is inside the critical range ${ }^{2}$ of the $l$-th PU, $v_{l}$, hence the CAP depends on the $l$-th PU activity probability. Due to the mobility of PU, the $\mathrm{CU}$ is outside the critical range at time $t$, therefore, the channel availability is independent whether the $l$-th PU is active or not. Mobility can thus change the dependency of CUs on the activity of existing PUs, leading to the time-variant CAP for each CU. In mobile scenarios, the CAP is time variant as shown and analyzed in [1] and a channel selection strategy based on this time-varying CAP has been proposed in [2]. ${ }^{3}$ Another impact of mobility in CRNs is link diversity, which leads to link-variant CAP. For example, in Fig. 1 (b), we note that, at time $t$, the $i$-th and $j$-th CUs $u_{i}$ and $u_{j}$ are outside the critical range of any $\mathrm{PU}$, but the $k$-th $\mathrm{CU} u_{k}$ is inside the critical range. In this case, the channel availability

\footnotetext{
${ }^{1}$ Herein, the notion "link" refers to connection between a pair of communicating users.

${ }^{2}$ The CUs are able to detect active PUs within a range, referred to as critical range, determined by the $\mathrm{PU}$ transmission range and by the $\mathrm{CU}$ interference range $[5,6]$. This range might vary for each CU especially in heterogeneous networks.

${ }^{3}$ See Section 2 for further discussion.
} 


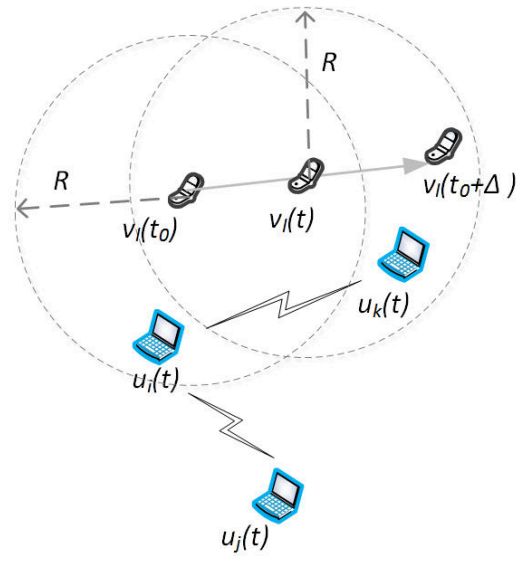

(a) CAP is not only time variant but also link varianr

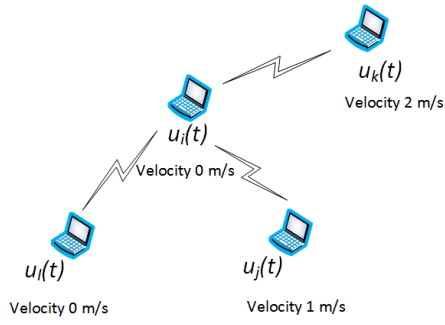

(b) CQ measurement is also link variant

Figure 1: Channel availability and channel quality are time and link variant in MCRNs.

for the link between $u_{i}$ and $u_{j}$ does not depend on the PU activity probability whereas the channel availability for link between $u_{i}$ and $u_{k}$, depends on the PU activity probability.

The availability of a channel for communication between a pair of CUs does not directly imply that the channel is of sufficient quality for data transmission. If the two nodes are communicating and if either one or both of them is/are mobile, then their CQ will be time variant. This is particularly due to the variation of relative distances between the nodes and changing of scattering objects in the environment, which lead to time-varying attenuation due to path loss and channel fading, respectively. Using the same reasoning as the linkvariant $\mathrm{CAP}$, the $\mathrm{CQ}$ also varies from one link to another due to a diverse mobility pattern. It is therefore crucial to incorporate both the time-varying $\mathrm{CAP}$ and $\mathrm{CQ}$ parameters to design a channel selection strategy that aims to maximize the rate of reliable data delivery.

In this paper, we propose a mobility-aware channel selection technique by jointly accounting for the parameters of time- and link-variant CAP and CQ for MCRNs. Underpinning this novel technique, the contributions of this work include the following.

i) We derive link-based CAP (L-CAP) estimation based on the relative distances among PUs and CUs in a given temporal interval;

ii) We obtain a mathematical expression that captures estimation of the linkbased CQ (L-CQ) in mobile scenarios;

iii) We propose a Link-oriented Channel-Availability and channel-Quality (LCAQ) based channel selection strategy that aims to maximize the link throughput by taking into account two prominent channel selection metrics, namely L-CAP and L-CQ, which are governed by the non-stationarity of the network topology induced by the user mobility; 


\begin{tabular}{|c|c|}
\hline Acronym & Full Name \\
\hline CRNs & Cognitive Radio Networks \\
\hline CU & Cognitive User \\
\hline PUs & Primary Users \\
\hline CAP & Channel Availability Probability \\
\hline CQ & Channel Quality \\
\hline SCRNs & Static Congitive Radio Networks \\
\hline MCRNs & Mobile Congitive Radio Networks \\
\hline L-CAP & Link-Channel Avaliblity Probability \\
\hline L-CQ & Link-Channel Quality \\
\hline L-CAP & Link-Channel Avalibility Probability \\
\hline RWPM & Random WayPoint Mobility \\
\hline LUP & Location Update period \\
\hline
\end{tabular}

Table 1: List of frequently-used acronyms.

iv) We demonstrate the benefits of the proposed strategy using numerical simulation for MCRNs.

The rest of the paper is organized as follows. For ease of reference, we provide the list of frequently-used acronyms in Table 1. In Section 2, we critically review and discuss related works. In Section 3, we state and formulate our approach to the problem of channel selection in MCRNs. In Section 4, we describe the network model of MCRNs. In Section 5, we present and discuss our main contribution of the L-CAQ strategy for channel selection. In Section 6, we evaluate the performance of this proposed scheme against the benchmark schemes through numerical simulation. Finally, Section 7 summarizes key points from this paper.

\section{Review of Existing Works}

Channel selection constitutes an important task for initiating communication in CRNs, which in turn is crucial for optimizing the network performance. Several works in the literature have emphasized the crucial roles of CAP and CQ in selecting an optimal channel from an available channel set. Most of the works, however, do not consider the impact of mobility on CRNs.

In [26], the authors proposed a method for channel availability analysis of the primary network by considering the Idle Probability of Consecutive Time Slots (IPCOTS) to be utilized in the sensing channel selection in CRNs. Based on discrete-time Markov model, the authors acquired the IPCOTS for every specific channel, according to a condition that its status in the primary network is distributed evenly. The sensing order of channel selection draws a suboptimal state-dependent policy, which is referred to as the IPCOTS policy. The channel analysis under the IPCOTS framework can provide extra information about the channel occupancy state in the primary network. Moreover, by using 
the IPCOTS policy the proposed channel selection technique can minimize the predicted number of channel switching to achieve the initial objective.

In [27], the authors exploited the spectrum hole and channel characteristics, and proposed a multi-channel selection algorithm to enable the prediction of spectrum hole in order to minimize the CU's interfering period to the primary network and boost channel utilization. This scheme has jointly considered the interference range and channel capacity in order to reduce the interference to primary network and improve the system performance. Through a simple amendment of statistical parameters over the channel quality, the scheme can achieve a higher throughput while reducing the collision rate.

In [22], the authors proposed an opportunistic sensing strategy, where the reward is adapted to the PU-link channel state information (CSI) prior to sensing. To achieve effective collision resolution, this strategy is integrated with a novel multi-channel first-come-first-served medium access control scheme. Both the individual and network throughputs were shown to improve by the aforementioned adaptive technique prior to sensing and randomization of sensing decisions.

Several channel selection techniques are presented in literature [28] - [37]. In [28], A channel selection technique was proposed using a fuzzy logic based decision making algorithm . In [35], a Markovian-based cooperative spectrum sensing policy was studied. In [29], the authors introduced a routing metric that aims to minimize the interference of the CUs to the PUs by estimating the channel availability through the channel history. In [30], the authors presented a channel selection algorithm based on the ON-OFF time distribution in cognitive radio networks. The channel selection algorithm aims to let the secondary user to discover the empty channel and reduce the rate of channel switching in an efficient way. Through observing and calculating the heavy-tailed OFF times for the primary users, the channel selection algorithm can show a significant decrease in channel switching rate and save the energy consumption.

As discussed in the above works, CAP is more prevalent for channel selection in static scenarios of the CRNs due to time-invariant CQ. In such a case, the selection problem can be projected as selecting a CAP entry (entries) from a weighted CAP vector where the weights are determined by fixed CQs within a set of available channels.

A limited number of works have addressed channel selection in the CRNs with mobility scenarios. In [3], the authors proposed an optimal routing metric for both static and mobile CRNs. However, consideration of the channel availability depends solely on the channel occupancy history, which is less accurate for MCRNs. In [1], a channel availability model was designed by taking into account the PU mobility. Considering the same model, a mobility aware channel selection technique is proposed in [2]. However, considering only the PU mobility is deemed insufficient to capture diverse possibilities of MCRNs where both the PU and CU may have non-stationary characteristics.

Pushing further these aforementioned works, in this paper we propose a channel selection technique that considers the impact of both PU and CU mobility. We first formulate the network model of MCRNs and establish precise 
mathematical expressions of the CAP and CQ that encapsulate time- and linkvariant characteristics of the network. Using these mathematical expressions, we then propose a channel selection algorithm and evaluate its performance against a number of benchmark schemes to demonstrate its superiority.

\section{Problem Statement}

When an arbitrary cognitive node $u_{i}$ wants to communicate with one of its neighbor nodes $u_{j}$, then $u_{i}$ needs to select the best channel among the list of licensed channels. A widely-used criterion for determining the "best channel" is the link throughput, $\psi_{i j}^{m}$ that corresponds to the amount of data per unit time that $u_{i}$ can successfully transmit to $u_{j}$ through an arbitrarily given channel $m$. At any given time $t$, based on the acquired values of CAP and CQ, user $u_{i}$ can approximate its link throughput to user $u_{j}$ at channel $m$ using the estimate

$$
\bar{\psi}_{i j}^{m}(t)=C A P_{i j}^{m}(t) \times C Q_{i j}^{m}(t) \times \psi^{m}
$$

where $C A P_{i j}^{m}(t) \in[0,1]$ is the channel availability probability for channel $m$ at time $t$ that equals to the PU inactive probabilities, denoted as $P_{o f f}^{m}(t)$; $C Q_{i j}^{m}(t) \in[0,1]$ is the (normalized) channel quality of channel $m$; and $\psi^{m}$ is the ideal link throughput for channel $m$, i.e., when $C A P_{i j}^{m}(t)=C Q_{i j}^{m}(t)=1$.

In static scenarios, the instantaneous values of the CAP and $C Q$ can be a priori known (when, e.g., the propagation environment and PUs' traffic are stationary) or simply estimated according to the channel occupancy and link quality history, respectively $[29,3]$. Throughout the rest of the paper, we refer to a method of estimating $\bar{\psi}_{i j}^{m}(t)$ via a priori available information or channel occupancy/quality history as the Traditional Method. It is customary that for the static scenarios with stationary environment and data traffic, both the $\mathrm{CAP}$ and CQ are time- and link-invariant (such that $C A P_{i j}^{m}(t)=C A P^{m}$ and $C Q_{i j}^{m}(t)=C Q^{m}$ ) and the link throughput solely depends on the activity of PUs across different network regions.

In mobile scenarios, the CAP and CQ are both time- and link-variant. Therefore, unlike the Traditional Method, the link throughput $\psi_{i j}^{m}(t)$ depends on three factors, namely: i) the Link-based $C A P^{m}(t)$, which will further depend on two factors, i.e., the PU inactive probabilities $P_{o f f}^{m}(t)$ and probability that a pair of CUs (e.g., $u_{i}$ and $u_{j}$ ) communication does not interfere with the active PUs, denoted as $N A_{i j}^{m}$; ii) Link-based $C Q_{i j}^{m}(t)$; and iii) ideal channel throughput $\psi^{m}$.

In order to clarify this issue, we consider an example shown in Fig. 2. The three PUs at time $t$ are $v_{l}(t), v_{n}(t)$ and $v_{m}(t)$ and they are active on channel $a$, $b$ and $c$, respectively. The three CUs at time $t$ who want to communicate one another are $u_{i}(t), u_{j}(t)$ and $u_{k}(t)$ and their critical range are $R_{i}, R_{j}$ and $R_{k}$, respectively.

For the sake of simplicity, we assume that the ideal channel throughput of channel $a, b$ and $c$ are $1 \mathrm{Mbps}$ and the channel quality for all the links at time $t$ are the same as depicted in Fig. 2. If we estimate the link throughput of link $u_{i} \leftrightarrow u_{j}$ and $u_{i} \leftrightarrow u_{k}$ at time $t$ for each channel using the Traditional Method, 


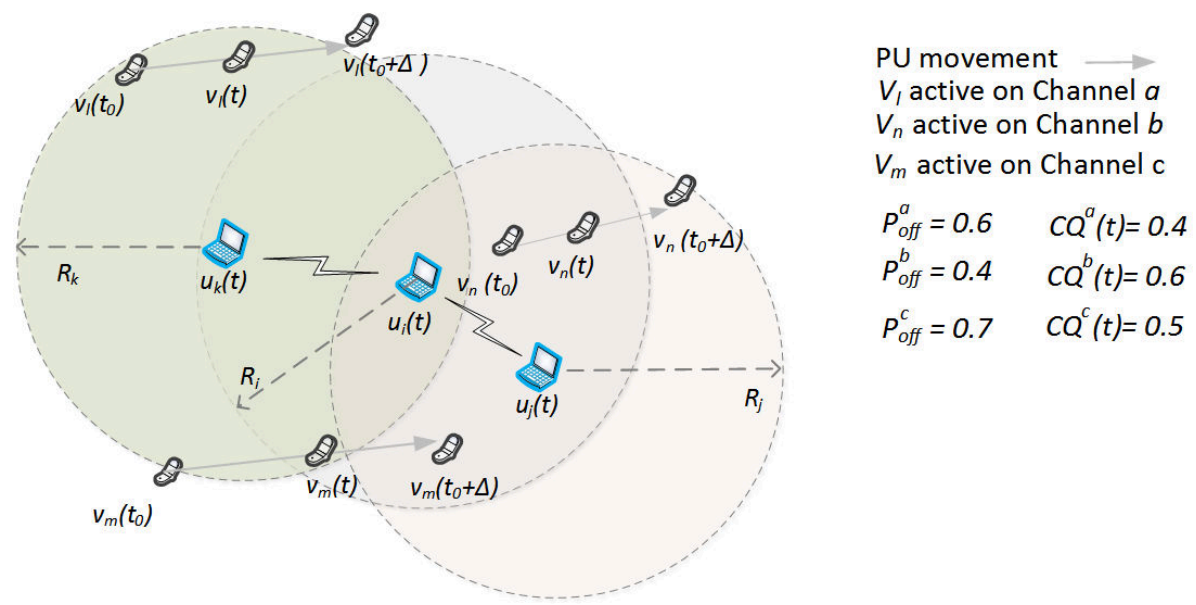

Figure 2: Channel selection in MCRNs.

then for both the links, we have $\bar{\psi}_{i j}^{a}(t)=\bar{\psi}_{i k}^{a}(t)=0.24 \mathrm{Mbps}, \bar{\psi}_{i j}^{b}(t)=\bar{\psi}_{i k}^{b}(t)$ $=0.24 \mathrm{Mbps}$ and $\bar{\psi}_{i j}^{c}(t)=\bar{\psi}_{i k}^{c}(t)=0.35 \mathrm{Mbps}$. The best channel at time $t$ according to this method is channel $c$. On the other hand, if we estimate the link throughput at time $t$ for each channel considering the mobility of users, we have that:

- For link $u_{i} \leftrightarrow u_{j}: \bar{\psi}_{i j}^{a}(t)=\left[P_{o f f}^{a}+\left(1-P_{o f f}^{a}\right) N A_{i j}^{a}\right] \times C Q_{i j}^{m}(t) \times \psi^{a}=0.4$ Mbps, $\bar{\psi}_{i j}^{b}(t)=0.24 \mathrm{Mbps}$ and $\bar{\psi}_{i j}^{c}(t)=0.35 \mathrm{Mbps}$

- For link $u_{i} \leftrightarrow u_{k}: \bar{\psi}_{i k}^{a}(t)=\left[P_{o f f}^{a}+\left(1-P_{o f f}^{a}\right) N A_{i k}^{a}\right] \times C Q_{i k}^{m}(t) \times \psi^{a}=0.24$ Mbps, $\bar{\psi}_{i k}^{b}(t)=0.6 \mathrm{Mbps}$ and $\bar{\psi}_{i k}^{c}(t)=0.35 \mathrm{Mbps}$.

According to this refined method, the best channel at time $t$ for both links $u_{i} \leftrightarrow u_{j}$ and $u_{i} \leftrightarrow u_{k}$ are channels $a$ and $b$, respectively, not channelc.

The aforementioned example demonstrates that in MCRNs, the CAP is not only time variant but also link variant, hence we refer to it as Link-based CAP (L-CAP). In addition to its time-variant nature due to changing distance and multipath propagation, the estimated channel equality will also be link-variant depending on the instantaneous distances of the CUs to the PUs. We therefore refer to this channel quality measure as Link-based Channel Quality (L-CQ) due to mobility variation of different CUs. We therefore envision to design a new channel selection strategy by exploiting both the L-CAP and L-CQ features that comprehensively capture the mobility characteristics of the network.

\section{Network Model}

We consider an MCRN where a set of CUs and PUs are mobile, denoted as $u_{i} \in U$ and $v_{l} \in V$, respectively. For the sake of simplicity, we assume that the CUs and PUs move according to the well-known Random WayPoint Mobility 


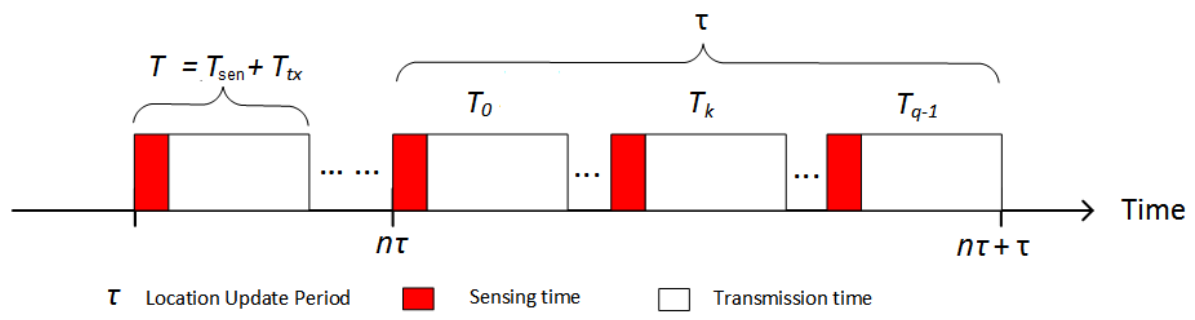

Figure 3: CU time organization related with location update interval (LUP).

(RWPM) model [11] inside a square network region $\mathcal{A}$. The vector $\mathbf{v}_{l}(t)$ denotes the two-dimensional position of the $l$-th PU at time instant $t$. The $l$-th PU traffic on the $m$-th channel is modeled as a two-state birth-death process $[5,12,13]$ with death rate $\alpha_{l, m}$ and birth rate $\beta_{l, m}$. In the on state, the $l$-th PU is active on channel $m$, and the active probability can be calculated using

$$
P_{l, m}^{o n}=\frac{\beta_{l, m}}{\left(\alpha_{l, m}+\beta_{l, m}\right)}
$$

whereas in the off state it is inactive on channel $m$ and the inactive probability can be computed using

$$
P_{l, m}^{o f f}=1-P_{l, m}^{o n} .
$$

The vector $\mathbf{u}_{i}(t)$ denotes the two-dimensional position of the $i$-th $\mathrm{PU}$ at time instant $t$. We consider more realistic PU spectrum occupancy models where different PUs roaming within the network region share the same channel [12].

As depicted in Fig 3, the CU activity is organized into fixed-sized frames with each having duration of $T$. Each frame interval $T$ is further divided into a sensing period $T_{\mathrm{sen}}[19,20]$, which measures the portion of the time slot assigned to the spectrum sensing, and a transmission period $T_{\mathrm{tx}}$, which measures the portion of the time slot devoted to the $\mathrm{CU}$ data transmission.

A pair of CUs can communicate with each other through licensed spectrum, which is organized in $N$ distinct bands/channels. More specifically, at time $t$, an arbitrary node $u_{i}$ communicates with another arbitrary node $u_{j}$ through a channel $m \in\{1, \ldots, N\}$. In order to select the best channel for communication, at time $t$, the $\mathrm{CU} u_{i}$ makes an attempt to estimate the link throughput $\psi_{i j}^{m}(t)$ for the link labelled by $e_{i j}^{m}$. This link throughput jointly accounts for two main metrics, namely L-CAP and L-CQ of the specified channel. These metrics are both time- and link-variant due to the mobility of PUs and CUs. As a consequence, in order to obtain the link throughput estimate $\bar{\psi}_{i j}^{m}(t)$, we assume that $\mathrm{CU} u_{i}$ is able to get the locations of its neighbors and the PUs periodically, which is governed by the Location Update period (LUP) $\tau$ as illustrated in Fig $3 .^{4}$

\footnotetext{
${ }^{4}$ It is reasonable to assume that the $\mathrm{CU}$ cannot obtain the locations of its neighbors and
} 


\section{L-CAQ based Channel Selection Technique in MCRNs}

In this section we analyze the mobility characteristics of the PUs and CUs to approximate the best channel that will enable communication between a pair of nodes. Since both the L-CAP and L-CQ are crucial for such a selection technique, we first discuss the estimation procedures of the L-CAP and L-CQ in mobile scenarios. We then discuss our proposed Link-oriented Channel-Availability and channel-Quality (L-CAQ) based channel selection technique for MCRNs.

\subsection{L-CAP Estimation in MCRNs}

As discussed in Section 4 and Fig. 3, the CU activity is organized into fixed-sized frames of $T$ duration, which is further divided into a sensing period $T_{\mathrm{sen}}$ and a transmission period $T_{\mathrm{tx}}$. We also assume that, each CU is able to update the locations of its neighbours and PUs every LUP $\tau=q T$. Due to the timing constraint of location acquisition, it is sensible to compute the L-CAP for every LUP. More specifically, with reference to Fig. 3, in order to estimate the L-CAP for a pair of nodes (e.g., $u_{i}$ and $u_{i}$ ) in the next temporal period, i.e., $[n \tau,(n \tau+\tau)), u_{i}$ has to take into account the impact of PUs activity on both nodes. For this purpose, we first precisely define the L-CAP parameters follows.

Definition 1 (L-CAP). Link-based CAP (L-CAP) is the probability of a channel licensed to $P U / P U$ s being available for the communication of a pair of neighbouring CUs over a given $L U P \tau$.

Computing the exact value of L-CAP for each LUP requires knowledge of the exact users positions at every frame $0, \ldots, q$, which is not directly available. Approximating these positions can be facilitated using the distance estimation procedure as outlined in Fig. 9 and detailed in Appendix 9.1. Based on the estimated distances among pairs of PUs and CUs, we obtain the probability of an arbitrary channel $m$ being available to CUs at frame $k \in\{0, \ldots, q-1\}$ as in the following proposition.

Proposition 1. At the time instant $t_{0}=n \tau$, the $i$-th $C U$ estimates the probability $p_{i j, m, \mathcal{L}}\left(T_{k}\right)$ of channel $m$ that is licensed to a set of PUs $\mathcal{L}$ being available for a pair of $i$-th and $j$-th $C U$ s in the next time slot $T_{k}=[n \tau+k T, n \tau+(k+1) T)$ for any $k \in\{0, \ldots, q-1\}$ as

$$
\tilde{p}_{i j, m, \mathcal{L}}\left(T_{k}\right)= \begin{cases}1, & \text { if }\left(\tilde{d}_{i, l}(t)>R_{i, l}\right) \operatorname{AND}\left(\tilde{d}_{j, l}(t)>R_{j, l}\right), \forall l \in \mathcal{L}, \\ \prod_{l \in \mathcal{L}} P_{l, m}^{o f f}, & \text { otherwise }\end{cases}
$$

PUs at every single time instant $t$ due to time-varying user locations in MCRNs and processing complexity. The PU location can be typically obtained through either location estimation algorithms or dedicated databases [18] while the CU location can be estimated either directly via dedicated positioning systems (e.g., GPS), or indirectly through location estimation algorithms [14]. Analysis of these localization techniques is beyond the scope of this paper. 
for all $t \in T_{k}$ where $R_{i, l}$ and $R_{j, l}$ denote the critical ranges of the $l$-th $P U$ with respect to $i$-th $C U$ and $j$-th $C U, \tilde{d}_{i, l}(t)$ and $\tilde{d}_{j, l}(t)$ denote the estimated distances between the $i$-th $C U$ and $l$-th $P U$, and the $j$-th $C U$ and $l$-th $P U$ at time $t \in T_{k}$, respectively. These estimated distances are all computed at time $t_{0}$.

Proof. The proof follows similar steps in [1] by modifying the distance estimation process among as discussed in Appendix 9.1 as well as considering the time- and link-varying nature of the connection between a pair of CUs $i$ and $j$.

The L-CAP metric for every LUP can then be expressed in the following proposition.

Proposition 2. At the time instant $t_{0}$, the $L$-CAP $C A P_{i j}^{m}(t)$ for $(i, j)$-th link for time interval $t \in[n \tau, n \tau+\tau)$ is given by the estimate of the probability of channel $m$ that is licensed to a set of PUs $\mathcal{L}$ being available in the next LUP $\tau=[n \tau, n \tau+\tau)$ as

$$
C A P_{i j}^{m}(t)=\frac{1}{q} \sum_{k=0}^{q-1} \tilde{p}_{i j, m, \mathcal{L}}\left(T_{k}\right)
$$

where $\tilde{p}_{i j, m, \mathcal{L}}\left(T_{k}\right)$ has been given in equation (4).

Proof. This L-CAP expression follows from averaging $\tilde{p}_{i j, m, \mathcal{L}}\left(T_{k}\right)$ given in Proposition 1 over all frames $k=0, \ldots, q-1$ within the LUP.

\subsection{L-CQ Estimation in $M C R N s$}

Consider two CUs $u_{i}$ and $u_{j}$ separated by distance $d_{i, j}$ that communicate over an arbitrary channel $m$ with bandwidth $W$. The channel quality of the link between $u_{i}$ and $u_{j}$ can be associated with the amount of data per unit time (in bits/s) that can be reliably transmitted over the given channel. Such a reliable transmission rate depends on not only channel characteristics, but also transceiver processing including coding and modulation techniques.

For a given specific modulation signal set $\mathcal{X}$ that may represent practical modulation techniques such as phase-shift keying (PSK), quadrature amplitude modulation (QAM) and pulse-amplitude modulation (PAM), the ultimate data rate for wireless information transfer over bandwidth $W$ can be well characterized by the instantaneous mutual information [21]

$$
\begin{aligned}
& I\left(\mathrm{SNR}_{m}, g_{m}\right)= \\
& W \cdot\left(\log _{2}|\mathcal{X}|-\frac{1}{|\mathcal{X}|} \sum_{x \in \mathcal{X}} \mathrm{E}\left[\log _{2} \sum_{x^{\prime} \in \mathcal{X}} e^{-\left|g_{m} \sqrt{\operatorname{SNR}_{m}}\left(x-x^{\prime}\right)+Z\right|^{2}+|Z|^{2}}\right]\right)
\end{aligned}
$$

where the expectation $\mathrm{E}[\cdot]$ is evaluated over $Z \sim \mathcal{N}_{\mathbb{C}}(0,1)$. Herein $|\mathcal{X}|$ denotes the cardinality of $\mathcal{X}, g_{m}$ is the complex-valued small-scale fading gain at channel $m$ and $\mathrm{SNR}_{m}$ denotes the signal power to noise ratio

$$
\mathrm{SNR}_{m} \triangleq \frac{P_{m}^{\mathrm{rx}}}{N_{0} W},
$$


where $P_{m}^{\text {rx }}$ is the received signal power (watt) at channel $m$ and $N_{0}$ is the noise spectral density (watt/Hz).

Mobility of users leads to variation in both the small-scale fading gain $g_{m}$ and the strength of $P_{m}^{\mathrm{rx}}$ that is determined by the large-scale fading gain. The time-variation of $g_{m}$ is mainly due to the change of scattering configuration can usually be captured by Rayleigh fading distribution in the rich scattering environments [23]. The time variation $P_{m}^{\mathrm{rx}}$ can be captured by linking its value with the transmit power $P^{\mathrm{tx}}$ as $[23]$

$$
P_{m}^{\mathrm{rx}}=P^{\mathrm{tx}} \times C_{0} \times f_{m}^{-2} \times d_{i, j}^{-2}
$$

where $C_{0} \times f_{m}^{-2} \times d_{i, j}^{-2}$ characterizes the path loss at a distance $d_{i, j}$ and carrier frequency $f_{m}$ of channel $m$ for some positive constant $C_{0}$. It is clear from (8) that unlike static scenarios, $P_{m}^{\mathrm{rx}}$ varies with time due to variation in distance $d_{i, j}$ and directly depends on the operating frequency of the given channel $m$.

It is widely understood that (see, e.g., [23]) temporal magnitudes of the small- and large-scale fading vary at different rates of change. Small-scale fading $g_{m}$ rapidly varies within a small period of time (short distance) whereas largescale fading (path loss) fluctuates over a longer travel distance. We can thus reasonably assume for a mobile communication with frame structure in Fig. 3 and certain mobile speeds that the large-scale fading changes every $T_{k}=T$ and the small-scale fading changes $B$ times for each $T_{k}, k=0, \ldots, q-1$. Therefore, the maximum data rate that can be reliably transmitted over a single sensing frame is given by the average mutual information of the block-fading channel with block size $B$, namely

$$
R_{i, j}\left(\mathrm{SNR}_{m ; k}, \boldsymbol{g}_{m, k}\right)=\frac{1}{B} \sum_{b=1}^{B} I\left(\mathrm{SNR}_{m ; k}, g_{m ; k, b}\right)
$$

where $I(\cdot, \cdot)$ has been defined in $(6)$ and $\mathrm{SNR}_{m ; k}=P_{m}^{\mathrm{rx}}(k) /\left(N_{0} W\right)$ is the effective receive SNR at frame time $T_{k}$, which depends on

$$
P_{m}^{\mathrm{rx}}(k)=P^{\mathrm{tx}} \times C_{0} \times f_{m}^{-2} \times d_{i, j}^{-2}\left(T_{k}\right),
$$

where $d_{i, j}\left(T_{k}\right)$ is the distance between CUs $i$ and $j$ at frame time $T_{k} \cdot{ }^{5}$ In equation (9), we have also defined $\boldsymbol{g}_{m ; k} \triangleq\left[g_{m ; k, 1}, \ldots, g_{m ; k, B}\right]$ as the $B$ realizations of the small-scale fading over time $T_{k}$. Note that the block size $B$ in equation (9) is closely related to the vehicle speed as each fading block $b \in\{1, \ldots, B\}$ can be defined by the coherence time of the channel, which is inversely proportional with the relative speed between two communicating devices [21, 24]. The faster the relative mobility, the value of $B$ will be larger (i.e., channel changes quickly within a frame) and vice-versa.

\footnotetext{
${ }^{5}$ Herein we assume that the distance between $(i, j)$-th CUs is approximately the same for a single frame of duration $T$.
} 
The average mutual information (9) well characterizes the instantaneous reliable transmission rate supported by the channel, taking into account the mobility of devices that induces fading. Based on this metric, we can define the L-CQ as follows.

Definition 2 (L-CQ). The link-based $C Q$ (L-CQ) per $L U P \tau=q T$ is defined by the average (normalized) mutual information over a period $\left[t_{0}, t_{0}+\tau\right]$ where $t_{0}$ is the starting time of the LUP.

A pair of communicating CUs $u_{i}$ and $u_{j}$ can then approximate their L-CQs over all unoccupied channels by estimating their corresponding channel states (based on the model in [25]) and distance, and calculating the average mutual information. Approximation of their L-CQ for channel $m$ is discussed in the following.

As discussed in Section 5.1, each CU is able to accurately inform its neighbors and PUs locations at time $t_{0}$, namely the beginning of LUP $\tau=q T$. Based on a predefined mobility pattern and initial positions at time $t_{0}$, a communicating pair of $(i, j)$-th CUs can estimate their distance $\tilde{d}_{i, j}\left(t_{0}+k T\right)$ for each transmission frame $k=0, \ldots, q-1$ within a LUP (see Appendix 9.2). Based on this estimated distance, we can then estimate the effective receive SNR for frame $k$ as

$$
\mathrm{SNR}_{m ; k}=\frac{P^{\mathrm{tx}} \times C_{0} \times f_{m}^{-2} \times \tilde{d}_{i, j}^{-2}\left(t_{0}+k T\right)}{N_{0} W} .
$$

In addition to this SNR estimate, each CU is assumed to be capable of acquiring small-scale fading information during the sensing time for each frame (cf. Fig 3) through pilot-aided channel estimation [38]. At frame $k$ and fading block (coherence time) $b$, this estimation yields noisy channel estimate for channel $m$, which is a function of the actual fading $g_{m ; k, b}[25]$

$$
\tilde{g}_{m ; k, b}=g_{m ; k, b}+e_{m ; k, b}
$$

where $e_{m ; k, b}$ is the channel state error term that is distributed according to $\mathcal{N}_{\mathbb{C}}(0, \epsilon)$ for some error variance $\epsilon>0$.

In a single LUP, the CUs $u_{i}$ and $u_{j}$ can then approximate their L-CQ at channel $m$ by computing the average mutual information of (9) using the estimated values $\mathrm{SNR}_{k}$ and $\tilde{g}_{k, b ; m}$. By accounting for the effective time for data transmission, we state our L-CQ metric to compute the link quality in the following proposition.

Proposition 3 (L-CQ). The $L-C Q$ at channel $m, C Q_{i j}^{m}(t) \in[0,1]$, where $t \in$ $\left[t_{0}, t_{0}+\tau\right]$ is given by the following expression

$$
C Q_{i j}^{m}(t)=\frac{T-T_{\text {sen }}}{T \times W \times \log _{2}|\mathcal{X}|} \cdot \frac{1}{q} \sum_{k=0}^{q-1} R_{i, j}\left(\mathrm{SNR}_{m ; k}, \tilde{\boldsymbol{g}}_{m ; k}\right)
$$

where $T$ and $T_{\text {sen }}$ are single frame time and sensing time, respectively (cf. Fig. 3), $W$ is the bandwidth of channel $m, \mathcal{X}$ is the modulation signal set and $q$ is the 
number of frames per location update interval. In the above $(13), R_{i, j}\left(\mathrm{SN} \mathrm{R}_{k}, \tilde{\boldsymbol{g}}_{k}\right)$ is the computed rate per frame (9) estimated using $\mathrm{SN}_{m ; k}$ in (11) and $\tilde{\boldsymbol{g}}_{m ; k}=$ $\left[\tilde{g}_{m ; k, 1}, \ldots, \tilde{g}_{m ; k, B}\right]$ where $g_{m ; k, b}$ has been given in $(12)$.

Remark 1. Here the estimated $\mathrm{SN}_{m ; k}$ is time variant because of its one of the key parameters $\tilde{d}_{i, j}$ is time variant. The estimation process of $\tilde{d}_{i, j}$ at time $t$ is discussed in Appendix 9.2.

\subsection{Proposed L-CAQ Channel Selection}

In the following we describe our proposed Link-oriented Channel-Availability and channel-Quality (L-CAQ) based channel selection strategy for MCRNs. In order to understand the concept of L-CAQ, we first define the term efficient channel, which is required for optimizing communication between a pair of CUs.

Definition 3 (Efficient Channel). An arbitrary channel $m$ is said to be an efficient channel that is chosen by an arbitrary $C U u_{i}$ to communicate with its neighbour $u_{j}$ during the period $[n \tau, n \tau+\tau)$, if the estimated link throughput $\bar{\psi}_{i j}^{m}(t)$ of channel $m$ is the highest compared to those of other available channels.

Note that estimation of the link throughput $\bar{\psi}_{i j}^{m}(t)$ is performed within the interval $[n \tau, n \tau+\tau), n \in \mathbb{R}^{+}$by taking into account the estimated L-CAP in Proposition 2 and estimated L-CQ in Proposition 3. The L-CAQ strategy then selects the efficient channel from the list of available channels as formalized in the following proposition.

Proposition 4. At the time instant $n \tau$, the $i$-th $C U u_{i}$ selects an efficient channel $m_{\text {eff }}^{i j}(n \tau)$ based on the estimated link throughput, $\bar{\psi}_{i, j}^{m}(\tau)$ in order to communicate with its neighbour $u_{j}$ by jointly incorporating the estimated $L$ $C A P$ and $L-C Q$ during the interval $\tau=[n \tau, n \tau+\tau)$ according to equation (1), i.e.,

$$
m_{\text {eff }}^{i j}(n \tau)=\arg \max _{m} \bar{\psi}_{i, j}^{m}(\tau)=\arg \max _{m} C A P_{i j}^{m}(n \tau) \cdot C Q_{i j}^{m}(n \tau) \cdot \psi^{m}
$$

where $C A P_{i j}^{m}(\cdot)$ and $C Q_{i j}^{m}(\cdot)$ have been given in Propositions 2 and 3, respectively, and $\psi^{m}$ is the ideal link throughput of channel $m$.

In the following section we demonstrate that by exploiting the dynamic variation of the L-CAP and L-CQ caused by the PUs and CUs mobility, the proposed L-CAQ is able to outperform the existing methods. The simulation results highlight the benefits of using the proposed technique for channel selection in the presence of mobile PUs and CUs.

\section{Simulation Results}

In this section we discuss several numerical experiments to prove the effectiveness of both the L-CAP and L-CQ as metrics that are closely associated with the channel throughput as well as evaluate the proposed L-CAQ based channel selection technique in MCRNs for establishing communication between a pair of CUs. We numerically measure the performance of L-CAP, L-CQ and L-CAQ through $10^{6}$ Monte Carlo runs. 


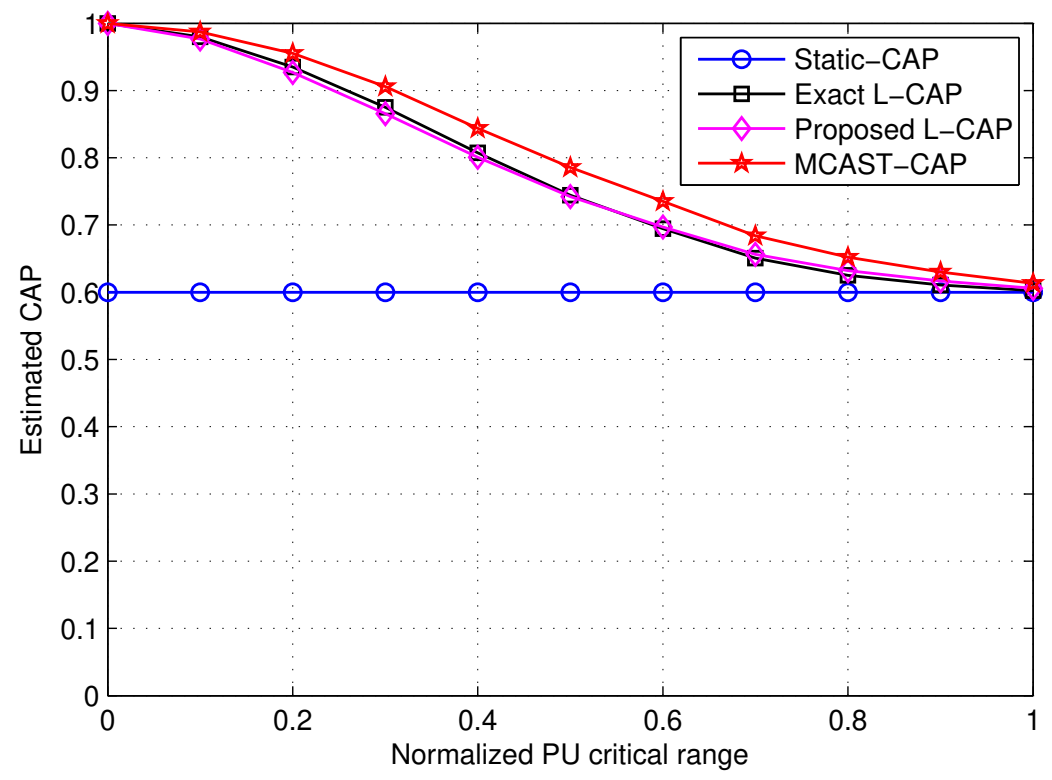

Figure 4: Estimated CAP versus PU critical range.

\subsection{Effectiveness of $L-C A P$}

In the following we demonstrate the effectiveness of our proposed L-CAP estimation (i.e., the estimated probability of a channel licensed to PU/PUs being available for the communication of a pair of neighboring CUs over a given interval) compared to the existing methods. Specifically, we consider four methods for estimating the CAP, namely : i) static estimation (i.e., based on only PU activity [29]), ii) Mobility-aware Channel-Availability probability based Channel Selection Technique (MCAST) [1], [2], iii) Proposed L-CAP with perfect knowledge of distance between $i$-th CU and $l$-th PU at time $t$, and iv) Proposed L-CAP with estimated distance between $i$-th CU and $l$-th PU at time $t$ as obtained in Proposition 1. Both PUs and CUs move in a network region with area of $1000 \mathrm{~m}^{2}$ according to the RWPM model with velocity uniformly distributed in the interval $[5,10] \mathrm{m} / \mathrm{s}$. The considered simulation set is as follow: sensing time $T_{\text {sen }}=1 \mathrm{~s}$, transmission time $T_{t x}=3 \mathrm{~s}$, location update period $\tau=20$, $\mathrm{PU}$ inactive probability $=0.6$.

In Fig. 4, we analyze CAP by varying the normalized PU critical range. We can observe that CAP estimation based on the PU inactive probability, referred to as Static-CAP, does not depend on the PU critical range. In contrast, the other three estimation methods are significantly affected by the PU critical range. This is so because all these three methods are designed by taking into account the mobile nature of the network. Apart from the Static-CAP method, we notice that the estimated CAP value decreases while the PU critical 


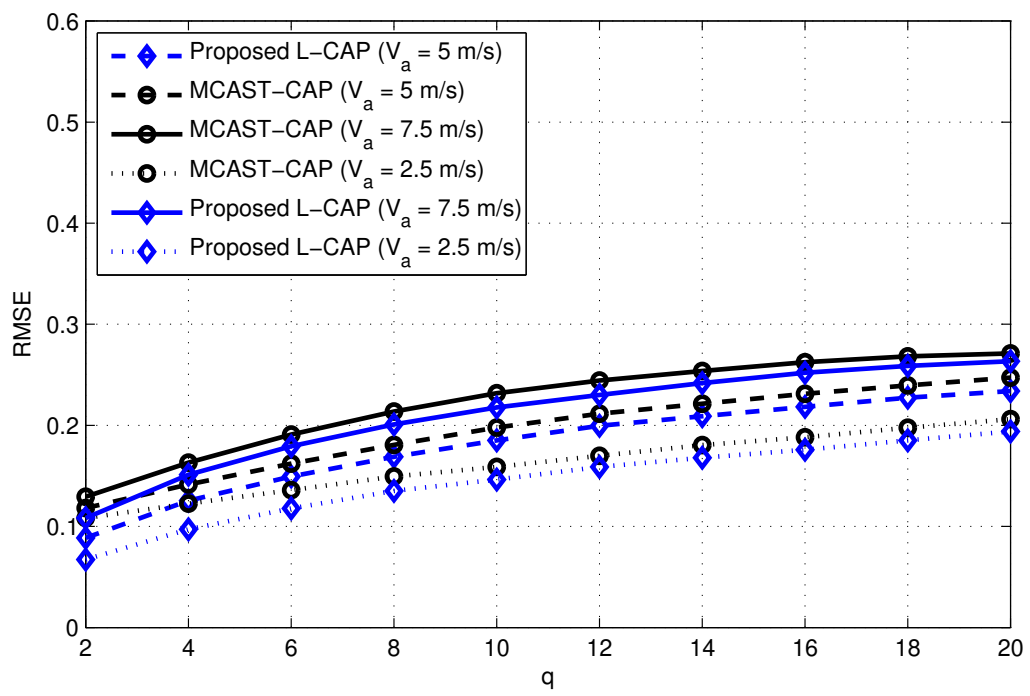

Figure 5: RMSE versus $q$ for different mean velocities. Note that $q$ herein is used to define variation in the LUP $\tau=q T$.

range increases. When all the network area is covered by the PU critical range (i.e., when the critical range $=1$ ), then all four estimation methods produce similar CAP values. We further notice that our proposed L-CAP estimation method provides less error compared to the other mobility-aware CAP estimation method, namely MCAST-CAP that was proposed in [1], [2]. This is sensible because the proposed method takes into account the link-oriented CAP rather than the node-based CAP, as captured by Proposition 2 .

The impact of localization error on CAP estimation is exhibited in Figs. 5 and 6 by adopting a similar simulation set to Fig. 4. There is an inherent trade-off between the network overhead and estimation error, i.e., the larger the time interval of the LUP $\tau$ is, the lower will the PU and CU position update rate be. Nevertheless, the larger $\tau$ causes a higher distance estimation error (see Appendix 9.1) that can be assessed by means of Root Mean Squared Error (RMSE). The estimation error can be further affected if the velocities of the PU and $\mathrm{CU}$ increase and/or the relative distance of the CUs for each link increases. In both cases, however, our proposed L-CAP estimation has comparatively less RMSE values than the MCAST-CAP, as shown in Figs. 5 and 6, respectively. This is so because the MCAST-CAP does not fully consider both the PU and $\mathrm{CU}$ mobility in the distance estimation procedure.

\subsection{Effectiveness of $L-C Q$}

The proposed L-CQ measure in Proposition 3 mainly depends on the accurate estimation of the received SNR and small-scale fading parameter. In Fig. 7, assuming no localization error (the computed distance is accurate), we verify the 


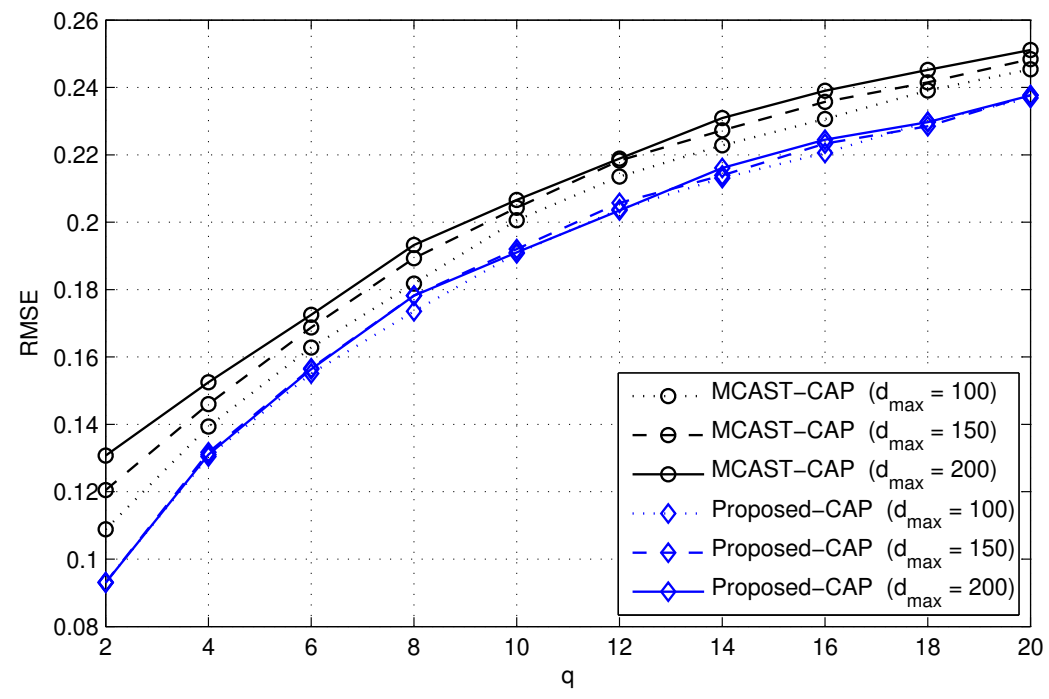

Figure 6: RMSE versus $q$ for different $d_{\max }$ (i.e., the maximum allowable distance between a pair of CUs to establish communication). Note that $q$ herein is used to define variation in the LUP $\tau=q T$.

sensitivity of L-CQ with respect to variation in distance and carrier frequency. As captured by equation (10), the link quality decreases exponentially with the increases in both distance and carrier frequency. A larger distance implies a significant attenuation in transmit signal power and a higher frequency leads to a shorter transmission range.

The small-scale fading parameter represents rapid-fluctuation (in the order of milliseconds) of transmit signal due to Doppler-induced mobility. The error in fading estimation (12) is directly proportional to the Doppler/terminal speed [38]. In order to maintain the same level of estimation accuracy, the time resources for channel estimation will need to increase linearly with the mobility speed, which lead to reduction of the amount of data that can be reliably transmitted. This latter point implies a lower L-CQ for a higher mobility speed.

\subsection{Effectiveness of $L-C A Q$}

In the following we demonstrate the effectiveness of our proposed L-CAQ method in Proposition 4 compared to the existing mobility-aware channel selection strategy, namely MCAST which selects the channel based on the maximum channel availability probability (MAX-CAP) metric. As argued in Sections 2 and 3, however, MAX-CAP may be insufficient in order to the select the best channel under mobile scenarios due to both the time- and link-variation of the channels. Instead, we envisage to select the best channel that has the highest link throughput $\psi_{i, j}^{m}(t)$. In practice the time- and link-variant nature of the 


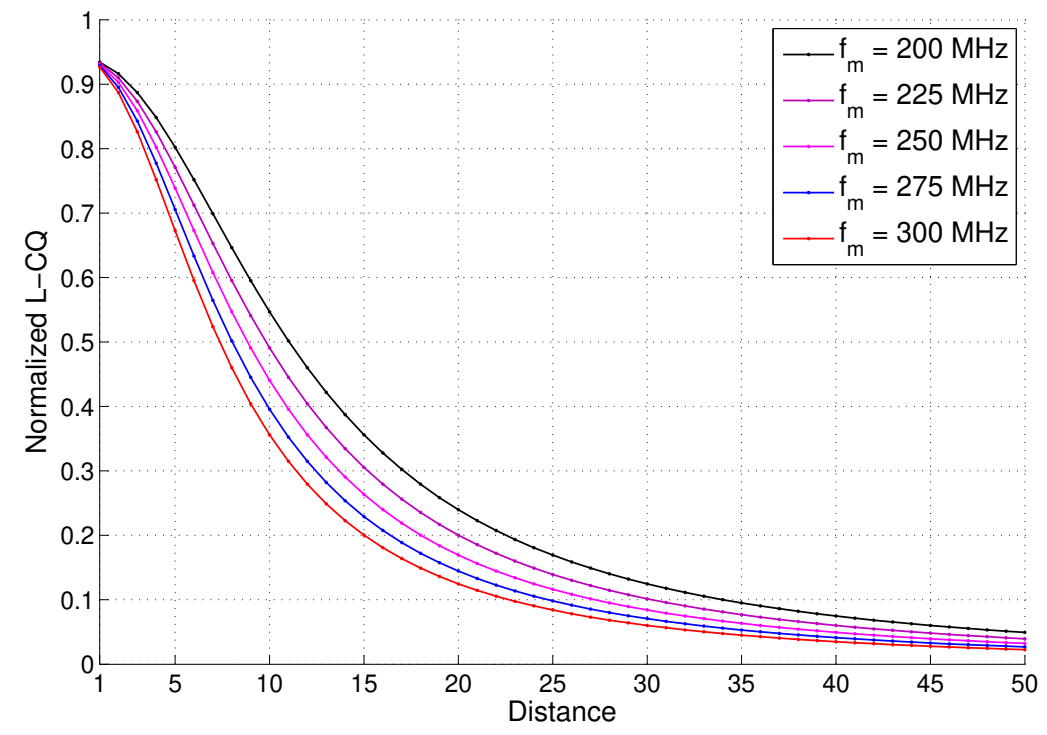

Figure 7: Normalized L-CQ vs Distance with the varying of channel frequency.

channel implies that computing the exact $\psi_{i}^{m}$ is challenging due to unavailability of required prior information. Our approach in this paper is to estimate the $\psi_{i, j}^{m}$ by individually estimating and subsequently multiplying the L-CAP and L-CQ values according to equation (1), which is possible through exploitation of LUP and sensing time per frame. In this way both the L-CAP and L-CQ are jointly taken into account when devising a channel selection strategy. For the purpose of comparison, we adopt the following simulation set: Number of PUs $=10$; number of channels $=5$ where 2 PUs are licensed on each channel, i.e., Multiple PUs for Channel [5]; PU inactive probability and ideal link throughput (ILT) for channels $\{1,2,3,4,5\}$ are given by $\{0.6,0.2,0.3,0.5,0.4\}$ and $\{2,2.5,5,7.5,10\}$, respectively; sensing time $T_{\text {sen }}=1 \mathrm{~s}$; transmission time $T_{\mathrm{tx}}=3 \mathrm{~s} ;$ and LUP $\tau=20$.

Four strategies for estimating the normalized link throughput (i.e., keeping the value of Link Throughput in between 0 to 1 ) are compared in Fig. 8, namely: i) Channel selection technique based on the highest ILT (labelled as MAX-ILT), ii) Channel selection technique in MCAST [1],[2], iii) Proposed channel selection technique with perfect knowledge of distance between $(i, j)$-th CUs and $l$-th PU at time $t$ (labelled as Exact), and iv) Proposed channel selection technique with proposed estimated distance between $(i, j)$-th CUs and $l$-th PU at time $t$ (labelled as Proposed). We note that the link throughput decreases with the increase of PU critical range in all the channel selection strategies due to a similar reasoning to that in Fig. 4. In the case of MCAST, the estimation error (i.e., its gap from the Exact method that indicates the actual link throughput) 


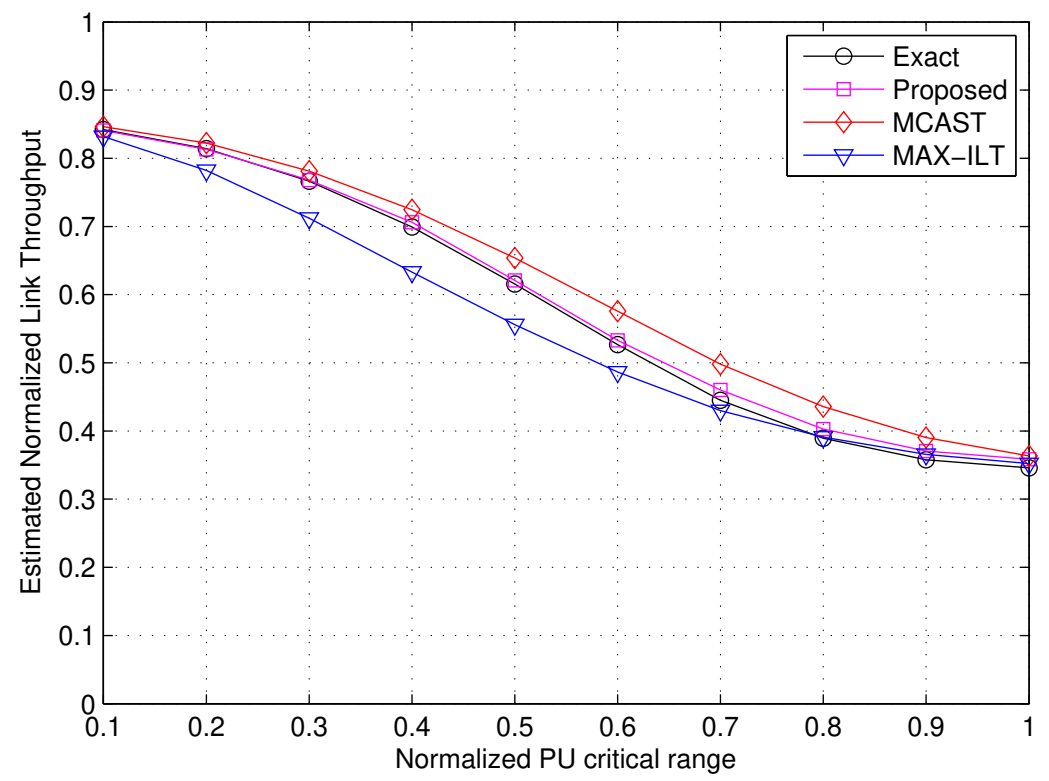

Figure 8: Estimated Normalized Link throughput versus PU critical range.

is large since it selects the channel according to only the node-based CAP. The $M A X-I L T$ method is neither accurate to capture the actual link throughput as the ILT criterion does not take into account the impact of users mobility to the time- and link-variant nature of the channel. On the other hand, joint consideration of both the L-CAP and L-CQ in the design of channel selection makes our proposed method attractive since it provides the most accurate link throughput estimate. This can be observed from a small gap between two curves in Fig. 8, namely the Exact and Proposed curves.

\section{Conclusion}

In this paper we have proposed a joint L-CAQ based channel selection technique for MCRNs. Based on the relative distances among PUs and CUs in a given temporal interval, we have first derived an estimate of the link-based channel availability probability (L-CAP). We have then obtained a mathematical expression that estimates the link-based channel quality (L-CQ) in mobile scenarios. By incorporating both the L-CAP and L-CQ metrics that are governed by the non-stationarity of the network topology in MCRNs, we have designed an effective channel selection strategy that maximizes the estimated link throughput. Numerical results have revealed the effectiveness of adopting the proposed scheme in MCRNs. 


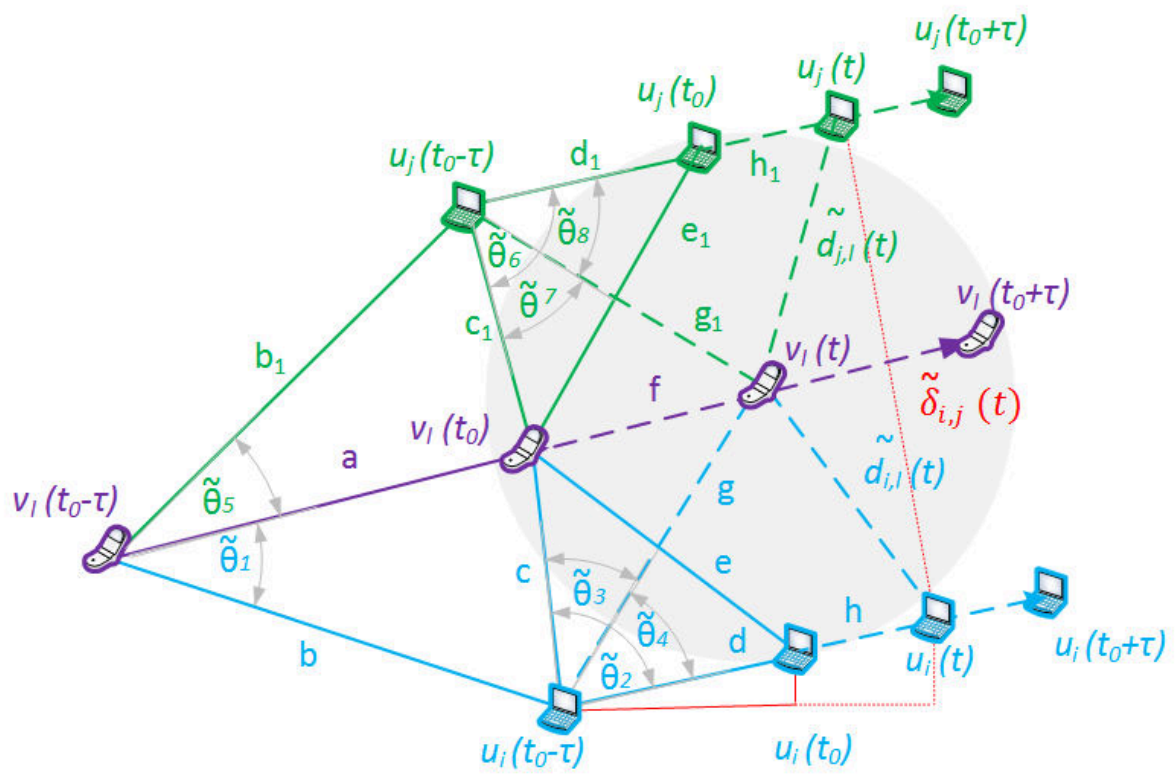

Figure 9: Distance estimation procedure.

\section{Acknowledgement}

The work of M. A. Rahman and K. Z. B. Zamli was supported in part by the University Malaysia Pahang (UMP)-RDU grant titled by "Palm Oil Supply Chain Traceability: Exploiting TV White Space". The work of A. T. Asyhari was supported in part by the UK Engineering and Physical Sciences Research Council (EPSRC) Global Challenge Research Fund-Cranfield Institutional Allocation under the project "RHENIUM: Reliable Heterogeneous IoT Networks for Indonesia Natural Disasters' Monitoring and Recovery Systems".

\section{Appendix}

\subsection{PU-CU Distance Estimation and Connection to Channel Availability}

At time $t_{0}=n \tau$, CUs $u_{i}$ and $u_{j}$ estimate the trajectory of PU $v_{l}$ for the time interval $\left[t_{0}, t_{0}+\tau\right)$ by exploiting to locations of these PU and CUs at time instant $t_{0}-\tau$ and $t_{0}$ and assuming that the PU does not change its direction in this interval. This assumption is reasonable because of the considered mobility model (RWMP) and the small value of the LUP $\tau$ [1].

The distance estimation procedure at time $t \in\left[t_{0}, t_{0}+\tau\right)$ is depicted in Fig. 9. At time $t, u_{i}$ estimates $\tilde{d}_{i, l}(t)$ by applying the law of cosine to the $\triangle$ : $u_{i}\left(t_{0}-\tau\right), u_{i}(t)$ and $v_{l}(t)$, i.e.,

$$
\tilde{d}_{i, l}(t)=\sqrt{g^{2}+(d+h)^{2}-2 g(d+h) \cos \left(\theta_{4}\right)}
$$


where

- $g$ can be achieved by applying the law of cosine to the $\triangle: u_{i}\left(t_{0}-\tau\right), v_{l}(t)$ and $v_{l}\left(t_{0}-\tau\right)$, i.e.,

$$
g=\sqrt{(a+f)^{2}+(b)^{2}-2(a+f) b \cos \left(\theta_{1}\right)}
$$

where $\theta_{1}=\cos ^{-1}\left(\frac{a^{2}+b^{2}-c^{2}}{2 a b}\right)$ and $f=a\left(t-t_{0}\right) / \tau$;

- $h=d\left(t-t_{0}\right) / \tau$;

- $\theta_{4}=\theta_{2}-\theta_{3}$ where $\theta_{2}$ and $\theta_{3}$ can be acquired from the $\triangle: u_{i}\left(t_{0}-\tau\right), v_{l}\left(t_{0}\right)$ and $u_{i}\left(t_{0}\right)$ as well as the $\triangle: u_{i}\left(t_{0}-\tau\right), v_{l}\left(t_{0}\right)$ and $v_{l}(t)$, respectively;

- $a, b, c, d$, and $e$ can be directly calculated using the locations information.

At time $t, u_{j}$ estimates $\tilde{d}_{j, l}(t)$ by applying the law of cosine to the $\triangle: u_{j}\left(t_{0}-\tau\right)$, $u_{j}(t)$ and $v_{l}(t)$, i.e.,

$$
\tilde{d}_{j, l}(t)=\sqrt{g_{1}^{2}+\left(d_{1}+h_{1}\right)^{2}-2 g_{1}\left(d_{1}+h_{1}\right) \cos \left(\theta_{8}\right)}
$$

where $a_{1}, b_{1}, c_{1}, d_{1}, e_{1}, f_{1}, g_{1}, h_{1}$ and $\theta_{8}$ can be obtained using similar steps to compute $g, h$ and $\theta_{4}$, respectively, as outlined for the case of $\tilde{d}_{i, l}(t)$ in equation (15).

After getting the estimated distances $\tilde{d}_{i, l}(t)$ and $\tilde{d}_{j, l}(t)$, if both $\tilde{d}_{i, l}(t)>R_{i, l}$ and $\tilde{d}_{j, l}(t)>R_{j, l}$, then the CUs $u_{i}$ and $u_{j}$ can use the channels licensed for the PU $v_{l}$ since both the CUs are outside the critical range of $v_{l}$ (i.e., $\tilde{p}_{i j, m, \mathcal{L}}\left(T_{k}\right)=1$ ), otherwise, $u_{i}$ and/or $u_{j}$ are inside the critical range of PU (i.e., $\left.\tilde{p}_{i j, m, \mathcal{L}}\left(T_{k}\right)=\prod_{l \in \mathcal{L}} P_{l, m}^{o f f}\right)$. This approach is sensible since it aligns with the policy of minimizing the interference on the PU transmission.

\subsection{Distance Estimation between a Pair of CUs}

The distance between CUs $i$ and $j$, i.e., $d_{i, j}(t)$, can be estimated by exploiting the CUs locations at time $t_{0}=n \tau$ and $t_{0}-\tau$ and assuming that the CUs do not change their direction within the interval $\left[t_{0}, t_{0}-\tau\right]$. This is reasonable for the same reasoning as in Appendix 9.1.

From Fig. 9, let us consider that at time $t_{0}$ and $t_{0}-\tau$, the exact locations of CUs $u_{i}$ and $u_{j}$ are given by $\left(u_{i}^{x}\left(t_{0}\right) ; u_{i}^{y}\left(t_{0}\right)\right)$ and $\left(u_{j}^{x}\left(t_{0}\right) ; u_{j}^{y}\left(t_{0}\right)\right)$, and $\left(u_{i}^{x}\left(t_{0}-\tau\right)\right.$; $\left.u_{i}^{y}\left(t_{0}-\tau\right)\right)$ and $\left(u_{j}^{x}\left(t_{0}-\tau\right) ; u_{j}^{y}\left(t_{0}-\tau\right)\right)$, respectively. If we know the two locations of node $u_{i}$ at two different time instants $t_{0}$ and $t_{0}-\tau$, we can then draw a RightAngle-Triangle, as shown in Fig. 9, and obtain the angle between hypotenuse and adjacent from the following equation, i.e.,

$$
\theta_{h, a}=\cos ^{-1}\left(\frac{u_{i}^{x}\left(t_{0}\right)-u_{i}^{x}\left(t_{0}-\tau\right)}{d}\right) .
$$

Since we assume that the CUs do not change their direction from $t_{0}-\tau$ to $t_{0}+\tau$, the new position of the $u_{i}$ at time $t$ can therefore be estimated as $u_{i}^{x}(t)$ 
$=\cos \theta_{h, a} \times(d+h)$ and $u_{i}^{y}(t)=\sin \theta_{h, a} \times(d+h)$. The position of $u_{j}$ at time $t$ can also be estimated by invoking the same steps. After approximating both the positions of CUs $u_{i}$ and $u_{j}$ at time $t$, the estimate of $d_{i, j}(t)$ can then be obtained by using the following equation

$$
\tilde{d}_{i, j}(t)=\sqrt{\left(u_{i}^{x}(t)-u_{j}^{x}(t)\right)^{2}+\left(u_{i}^{y}(t)-u_{j}^{y}(t)\right)^{2}} .
$$

\section{References}

[1] A.S. Cacciapuoti, M. Caleffi, L. Paura and M. A. Rahman, Channel availability for mobile cognitive radio networks. Journal of Network and Computer Applications, Volume 47, Pages 131-136, 2015.

[2] M. A. Rahman, R. Savoia and M. M. Uddin, MCAST: Mobility-aware channel-availability based channel selection technique. The Eleventh International Conference on Networking and Services, 2015.

[3] M. Caleffi, I. F. Akyildiz and L. Paura, OPERA: Optimal routing metric for cognitive radio ad hoc networks. IEEE Transactions on Wireless Communications, Volume 11, Issue no. 8, Pages 2884-2894, 2012.

[4] S. Abdelaziz and M. ElNainay, Metric-based taxonomy of routing protocols for cognitive radio ad hoc networks. Journal of Network and Computer Applications, Volume 40, Pages 151-163, 2014.

[5] A. S. Cacciapuoti, I. F. Akyildiz and L. Paura, Optimal primary-user mobility aware spectrum sensing design for cognitive radio networks. IEEE Journal on Selected Areas in Communications, Volume 31, Issue no. 11, Pages 2161-2172, 2013.

[6] A. Ghasemi and E. S. Sousa, Optimization of spectrum sensing for opportunistic spectrum access in cognitive radio networks. Proc. of IEEE Consumer Communications and Networking Conference (CCNC), 2007.

[7] S.C. Jha, U. Phuyal, M.M. Rashid and V.K. Bhargava, Design of OMCMAC: An opportunistic multi-channel MAC with QoS provisioning for distributed cognitive radio networks. IEEE Transactions on Wireless Communications, Volume 10, Issue no. 10, Pages 3414-3425, 2011.

[8] D. Xue, E. Ekici and X. Wang, Opportunistic periodic MAC protocol for cognitive radio networks. Proc. of IEEE Global Telecommunications Conference (GlobeCom), 2010.

[9] H. B. Salameh, O. S. Badarneh, Opportunistic medium access control for maximizing packet delivery rate in dynamic access networks. Journal of Network and Computer Applications, Volume 36, Pages 523-532, 2013. 
[10] A. C. Talay and D. T. Altilar, Self adaptive routing for dynamic spectrum access in cognitive radio networks. Journal of Network and Computer Applications, Volume 36, Pages 1140-1151, 2013.

[11] T. Camp, J. Boleng and V. Davies, A survey of mobility models for ad hoc network research. Wireless Communications and Mobile Computing, Volume 2, Issue no. 1, Pages 483-502, 2002.

[12] A. S. Cacciapuoti, I. F. Akyildiz and L. Paura, Primary-user mobility impact on spectrum sensing in cognitive radio networks. Proc. of IEEE Symposium on Personal, Indoor, Mobile and Radio Communications, 2011.

[13] Won-Yeol Lee and I.F. Akyildiz, Optimal spectrum sensing framework for cognitive radio networks. IEEE Transactions on Wireless Communications, Volume 7, Issue no. 10, Pages 3845-3857, 2008.

[14] S. Liu, Y. Chen, W. Trappe and L.J. Greenstein, Non-interactive localization of cognitive radios based on dynamic signal strength mapping. Proc. of IEEE International Conference on Wireless On-Demand Network Systems and Services (WONS), 2009.

[15] S. Parvin, F. K. Hussain, O. K. Hussain, S. Han and B. Tian, Cognitive radio network security: A survey. Journal of Network and Computer Applications, 2013.

[16] Z. Ning, Q. Song, Y. Huang and Lei Guo, A channel estimation based opportunistic scheduling scheme in wireless bidirectional networks. Journal of Network and Computer Applications, Volume 39, Pages 61-69, 2014.

[17] A. Avokh and G. Mirjalily, Interference-aware multicast and broadcast routing in wireless mesh networks using both rate and channel diversity. Computers and Electrical Engineering, Volume 40, Pages 614-640, 2014.

[18] M. Caleffi and A. S. Cacciapuoti, Optimal database access for TV white space. IEEE Transaction on Wireless Communications, Volume 64, Issue no. 1, Pages 83-93, 2016.

[19] A. S. Cacciapuoti, M. Caleffi, L. Paura and R. Savoia, Decision maker approaches for cooperative spectrum sensing: Participate or not participate in sensing? IEEE Transactions on Wireless Communications, Volume 12, 2013.

[20] A.S. Cacciapuoti, M. Caleffi, D. Izzo and L. Paura, Cooperative spectrum sensing techniques with temporal dispersive reporting channels. IEEE Transactions on Wireless Communications, Volume 10, 2011.

[21] A. Guillén i Fàbregas and G. Caire, Coded modulation in the block-fading channel: Coding theorems and code construction. IEEE Transactions on Information Theory, Volume 52, Issue no. 1, Pages 91-114, 2006. 
[22] Y. Lu, and A. Duel-Hallen, Channel-aware spectrum sensing and access for mobile cognitive radio ad hoc networks. IEEE Transactions on Vehicular Technology, Volume 65, Issue no. 4, Pages 2471-2480, 2016.

[23] T. S. Rappaport, Wireless Communications: Principles and Practice, 2nd ed. Pearson, 2010.

[24] A. T. Asyhari, T. Koch, and A. Guillén i Fàbregas, Nearest neighbor decoding and pilot-aided channel estimation for fading channels. arXiv: 1301.1223 [cs.IT], 2014.

[25] A. T. Asyhari and A. Guillén i Fàbregas, MIMO block-fading channels with mismatched CSI. IEEE Transactions on Information Theory, Volume 60, Issue no. 11, Pages 7166-7185, 2014.

[26] N. Jiang, J. Hua, Z. Shang and K. Yang, A new method for channel availability analysis and the associated policy design for selection of channel sensing order in CRNs. IEEE Transactions on Signal Processing, Volume 64, Issue no. 9, Pages 2443-2458, 2016.

[27] J. Lee and H. K. Park, Channel prediction-based channel allocation scheme for multichannel cognitive radio networks. Journal of Communications and Networks, Volume 16, Issue no. 2, Pages 209-216, 2014.

[28] Y. Yao, S. R. Ngoga, D. Erman and A. Popescu, Competition-based channel selection for cognitive radio networks. Proc. of IEEE Wireless Communications and Networking Conference (WCNC), Shanghai, Pages 1432-1437, 2012.

[29] K.R. Chowdhury and I.F. Akyildiz, CRP: A routing protocol for cognitive radio ad hoc networks. IEEE Journal of Selected Areas in Communications, Volume 29, Issue no. 4, Pages 794-804, 2011.

[30] S. Sengottuvelan, J. Ansari, P. Mhnen, T. G. Venkatesh, and M. Petrova, Channel selection algorithm for cognitive radio networks with heavy-tailed idle times, IEEE Transactions on Mobile Computing, Volume 16, Pages 1258-1271, 2017.

[31] X. Zhang, Y. Tan, C. Liang, Y. Li and J. Li, A Covert Channel over VoLTE via Adjusting Silence Periods, IEEE Access, 2018.

[32] L. Fan, X. Lei, N. Yang, T. Q. Duong, and G. K. Karagiannidis, Secure Multiple Amplify-and-Forward Relaying With Cochannel Interference, IEEE Journal of Selected Topics in Signal Processing, Volume. 10, Pages 1494-1505, 2016.

[33] M. Z. A. Bhuiyan, G. Wang, J. Wu, J. Cao, X. Liu, and T. Wang, Dependable Structural Health Monitoring Using Wireless Sensor Networks, IEEE Transactions on Dependable and Secure Computing, Volume 14, Pages 363-376, 2017. 
[34] T. Wang, J. Zenga, M. Z. A. Bhuiyan, Y. Chen, Y. Cai, H. Tian and M. Xie, Energy-efficient relay tracking with multiple mobile camera sensors, Computer Networks, Volume 133, Pages 130-140, 2018.

[35] S. Fazeli-Dehkordy, J. Abouei, K. N. Plataniotis and S. Pasupathy, Markovian-based framework for cooperative channel selection in cognitive radio networks. IET Communications, Volume 8, Issue no. 14, Pages 24582468, 2014.

[36] S. Sengottuvelan, J. Ansari, Petri Mahonen, T.G. Venkatesh and M. Petrova, Channel Selection Algorithm for Cognitive Radio Networks with Heavy-Tailed Idle Times. IEEE Transactions on Mobile Computing, Volume 16, 2017.

[37] Mohammed Hashem, Shrief I. Barakat and Mahmoud A. AttaAlla Enhanced tree routing protocols for multi-hop and multi-channel cognitive radio network (EMM-TRP). Journal of Network and Computer Applications, Volume 100, 2016.

[38] A. T. Asyhari and S. ten Brink, Orthogonal or superimposed pilots? A rateefficient channel estimation strategy for stationary MIMO fading channels. IEEE Transactions on Wireless Communications, Volume 16, Issue no. 5, Pages 2776-2789, 2017. 